\title{
Advances in the medical management of benign prostatic hyperplasia
}

\author{
Michael A.S. Jewett, Laurence H. Klotz, for the University of Toronto Uro-Oncology Program
}

$\infty$

See related article page $\mathrm{I} 853$

$\mathrm{P}$ rostatic disease eventually affects almost all men; benign prostatic hypertrophy or hyperplasia (BPH) is an inevitable part of aging. It has several effects, including progressive bothersome voiding symptoms (now termed lower urinary tract symptoms instead of prostatism) that trigger physician visits and costly treatment and may elevate patients' serum levels of prostate-specific antigen (PSA), leading to concern and diagnostic procedures to exclude prostate cancer. ${ }^{1}$ Two landmark randomized trials recently have seriously challenged the current treatment paradigm for managing $\mathrm{BPH}$. The implications of these study findings are important in the context of prostate disease prevention, detection and treatment.

Until the early ig9os, lower urinary tract symptoms were usually treated with transurethral resection of the prostate. However, as the natural history of BPH became better understood and the drugs available more selective, expectant management (for men with mild symptoms) and pharmacotherapy (moderate to severe symptoms) have been widely embraced. The benefits of symptomatic improvement, such as better urinary flow, symptom relief and improved quality of life, have been well documented in randomized clinical trials. ${ }^{2}$ Nevertheless, uncertainty has persisted about the effects of these therapies on the complications of $\mathrm{BPH}$, which include urinary retention, refractory hematuria, bladder calculi, recurrent urinary tract infections and renal failure. The Proscar Long-term Efficacy and Safety Study, a 4-year randomized trial of finasteride versus placebo, has for the first time demonstrated that the natural history of $\mathrm{BPH}$ could be altered by longterm therapy and acute urinary retention, prevented., ${ }^{3,4}$

The 2 major classes of drugs used to treat BPH are $\alpha$ adrenergic antagonists or $\alpha$-blockers (doxazosin, terazosin, tamsulosin and alfluzosin) and 5 - $\alpha$-reductase inhibitors (finasteride and dutasteride). Alpha-blockers relax the smooth muscle fibres of the bladder neck and prostate, thereby reducing the dynamic components of prostatic obstruction. Five$\alpha$-reductase inhibitors decrease levels of intracellular dihydrotestosterone (the major growth-stimulatory hormone in prostate cells) without reducing testosterone levels. This leads to prostatic size reduction of $20 \%-30 \% .{ }^{3}$ Symptom relief occurs within 2 weeks of initiating $\alpha$-blockers, compared with several months with finasteride.

The potential for synergy between these 2 classes of drugs has been an attractive hypothesis. Alpha-blockade would reduce the dynamic component of obstruction, and a $5^{-\alpha-}$ reductase inhibitor would reduce the fixed component. Recently, McConnell and colleagues reported the results of the landmark Medical Therapy of Prostatic Symptoms study. ${ }^{5}$ This long-term randomized trial compared the efficacy of doxazosin, finasteride and a combination of both drugs against placebo. The strengths of the trial were its large size $(n=3047)$ and objective end points. The use of the doxazosin either alone or in combination with finasteride retarded the clinical progression of $\mathrm{BPH}$ compared with placebo; the combination therapy was significantly more effective than either drug alone. At 5 years, the number needed to treat for each patient who avoided clinical progression was I2. Clinically significant side effects, mainly postural hypotension, were infrequent and not age-related; they led to cessation of therapy in $18 \%-27 \%$ of the men involved in the study. Higher serum concentrations of PSA and larger prostate volume correlated with the risk of progression. In summary, the Medical Therapy of Prostatic Symptoms study showed that BPH is a progressive disease; progression can be prevented by medical therapy; patients at risk for progression can be readily identified by PSA level, prostatic volume and symptom severity; and the combination of finasteride and doxazosin is more effective than either alone in preventing progression, particularly in high-risk groups.

It is well known that men with $\mathrm{BPH}$ can experience prostate cancer as well. A recent large study, the Prostate Cancer Prevention Trial, was designed to determine if primary prevention of prostate cancer is possible. ${ }^{6}$ The agent chosen, finasteride, was administered to men older than 55 years who were deemed to be at low risk of prostate cancer. Among the men randomly assigned to receive placebo, prostate cancer was diagnosed in $24.4 \%$ during the 7 years of the study, compared with I $8.4 \%$ of those who received finasteride: an absolute risk reduction of $6 \%$ and a relative risk reduction of $25 \%$. Side effects that occurred were minor and related mainly to sexual function.

These results are highly significant, clinically as well as statistically. Urinary symptoms among finasteride-treated patients were much improved and the overall risk of prostate cancer was reduced by $25 \%$ - a rate almost unheard of in the field of cancer prevention. Because PSA levels are reduced in men with $\mathrm{BPH}$ who are taking finasteride, rising PSA findings are more likely to be caused by prostate cancer. Taking this drug may therefore provide a diagnostic advantage, as well.

Remarkably, $25 \%$ of men in the placebo group were found to have prostate cancer when the systematic biopsies taken at study exit were evaluated. This high rate of cancer detection suggests that the method used in the study, transrectal ultrasound-guided prostate biopsy, detects clinically significant numbers of cancers irrespective of PSA levels. Since this rate of diagnosis is approximately io times the historic risk of death from prostate cancer, the fact that most of these cancers are indolent is indisputable. These findings are in sharp con- 
trast to previous reports of screening in the general male population, in which 10\%-15\% had an elevated PSA level and, of these, $35 \%$ ( $3 \%-5 \%$ of men in total) had diagnoses of cancer. ${ }^{7}$

Two large studies have demonstrated a preventive benefit from finasteride: the Medical Therapy of Prostatic Symptoms study showed a reduction in the rate of progression of $\mathrm{BPH}$, and the Prostate Cancer Prevention Trial showed a reduction in the rate of prostate cancer diagnosis. Should selected patients now be offered finesteride to lower their risk of developing prostate cancer and BPH progression? The answer, based on these trials, is unequivocally yes. Men with large prostate glands and lower urinary tract symptoms that are moderate to severe derive considerable benefit from finasteride; the rate reduction for prostate cancer is an added bonus. The cost of the drug (which might well be offset by reduced treatment) and a very mild decrease in sexual function seem to be the only drawbacks to its widespread use by men 55 years of age or older with no evidence of prostate cancer. Moreover, there is increasing recognition that PSA levels in the mildly elevated range ( $<$ Io $\mathrm{ng} / \mathrm{mL}$ ) reflect the presence and extent of BPH more than the presence of cancer. ${ }^{1}$

This article has been peer reviewed.

Michael Jewett is with the Ontario Cancer Institute, Princess Margaret Hospital. Laurence Klotz is with the Sunnybrook and Women's College Health Sciences Centre, Toronto, Ont.

Competing interests: None declared.
Contributors: All of the authors participated in the drafting and critical revision of this article, and approved the final version for publication.

\section{REFERENCES}

I. Stamey TA, Caldwell M, McNeal JE, et al. The prostate specific antigen era in the United States is over for prostate cancer: What happened in the last 20 years? JUrol 2004;172(4 Pt I):I297-30I.

2. Lepor H, Lowe FC. Evaluation and nonsurgical management of benign prostatic hyperplasia. In: Walsh PC, Retik AB, Vaughn E, et al, editors. Campbell's urology. Vol 2. 8th ed. Philadelphia: Saunders; 2002. p. I337-78.

3. Roehrborn CG, Boyle P, Bergner D, et al; Proscar Long-Term Efficacy and Safety Study (PLESS) Study Group. Serum prostate-specific antigen and prostate volume predict long-term changes in symptoms and flow rate: results of a four-year, randomized trial comparing finasteride versus placebo. Urology I999;54:662-9.

4. McConnell JD, Bruskewitz R, Walsh P, et al; Finasteride Long-Term Efficacy and Safety Study Group. The effect of finasteride on the risk of acute urinary retention and the need for surgical treatment among men with benign prostatic hyperplasia. N Engl J Med I998;338:557-63.

5. McConnell JD, Roehrborn CG, Bautista OM, et al. The long-term effect of doxazosin, finasteride, and combination therapy on the clinical progression of benign prostatic hyperplasia. N Engl J Med 2003;349:2387-98.

6. Thompson IM, Pauler DK, Goodman PJ, et al. Prevalence of prostate cancer among men with a prostate-specific antigen level $\leq 4.0 \mathrm{ng}$ per milliliter. $N$ Engl J Med 2004;350:2239-46.

7. Catalona WJ, Smith DS, Ratliff TL, et al. Measurement of prostate-specific antigen in serum as a screening test for prostate cancer. N Engl J Med I991;324:II56-6I.

Correspondence to: Dr. Michael Jewett, Ontario Cancer Institute, Princess Margaret Hospital, Rm. 3-124, 6ro University Ave., Toronto ON M5G 2M9; fax 4I6 340-4275; m.jewett@utoronto.ca

Members of the University of Toronto Uro-Oncology Program: Antonio Finelli, Neil Fleshner, Robert Nam, Sharon Sharir, John Trachtenberg and Alexander Zlotta 